\title{
LA DIMENSIÓN CONSTITUCIONAL DEL PODER PRESUPUESTARIO DE LA UNIÓN EUROPEA
}

\author{
Antonio-Martín Porras Gómez
}

Sumario: 1. Precisiones CONCEPTUALES EN tORnO Al CONSTitucioNALISMO SUPRANACIONAL. 2. LA PROGRAMACIÓN PRESUPUESTARIA EUROPEA. 2.1. El sistema de recursos propios. 2.2. Análisis del carácter supranacional del sistema de recursos propios. 3. CONCLUSIONES. 4. BIBLIOGRAFÍA.

\section{PRECISIONES CONCEPTUALES EN TORNO AL CONSTITUCIONALISMO SUPRANACIONAL}

El constitucionalismo supranacional hace referencia al sometimiento de formas de poder supranacional a estructuras, procesos, principios y valores constitucionales LoUgHLIN (2011: p. 47). Se articula en torno a dos categorías conceptuales, supranacionalidad y constitucionalismo, que se caracterizan por un alto nivel de abstracción. En primer lugar, hay que señalar que el concepto de supranacionalidad no resulta un concepto claramente definido. Hace referencia a una realidad relativamente reciente en la Historia, y por lo tanto su elaboración teórica ha corrido paralela a este retraso. Etimológicamente, si se hace una somera observación del término, nos encontramos con que se refiere a una realidad que se sitúa por encima de la nación.

La mención que la supranacionalidad hace a la nación genera una primera confusión. En efecto, nación es un concepto político en cierto modo preterido, y cuyo significado jurídico se limita a hacer referencia al sujeto detentador de la soberanía en determinados entes políticos de base democrática. Las características semánticas, tanto políticas como jurídicas, de la palabra nación, no se insertan bien en el concepto de supranacionalidad.

La supranacionalidad no hace referencia a la nación entendida como un grupo humano con una historia y una cultura propias, identificable en términos étnicos, religiosos, lingüísticos, históricos o geográficos (entendiendo nación 
en sentido cultural como etnos). La supranacionalidad tampoco hace referencia exactamente a la nación como sujeto soberano en entes políticos de base democrática (entendiendo nación en sentido jurídico como demos). Sin embargo, este sentido jurídico de nación como sustento de la soberanía en las formas de Estado democráticas apunta ya al auténtico significado que alberga el término supranacionalidad. En efecto, la supranacionalidad hace referencia a una realidad que se sitúa por encima del Estado, entendido éste como ente que ostenta el ejercicio de la soberanía. Es decir, que la supranacionalidad se referencia a la soberanía propia del Estado, independientemente de que esa soberanía estatal esté a su vez sustentada en una nación o no (puesto que puede sustentarse en otros elementos como un Rey, un Parlamento, un Partido, un Líder, un Orden divino... o en varias naciones, en el caso de un Estado multinacional). Consideramos por tanto que la nacionalidad en el sentido de supra, inter o subnacionalidad, se referencia al Estado soberano como punto de partida.

Lo cierto es que los vocablos de internacional, supranacional o subnacional se construyeron en una época en la que el modelo de Estado era el Estadonación. Esa época ha sido preterida en la actualidad, y por lo tanto toda indagación que se haga en torno al término de supranacionalidad ha de despojarse de la idea de Estado-nación para quedarse sólo con la idea de Estado. Por lo tanto, supranacionalidad hace referencia a elementos que se sitúan por encima del Estado. De la misma forma que internacionalidad hace referencia a elementos que se sitúan entre los Estados, y subnacionalidad a elementos por debajo de los Estados.

A este respecto, hay que señalar que el nivel internacional no constituye más que la superposición de los Estados. El nivel internacional corresponde simplemente al nivel de poder del Estado en su proyección ad extra. Hace referencia por tanto a estructuras de poder «pasivas», que se limitan a recoger un impulso político que viene de los Estados miembros ${ }^{1}$. El nivel supranacional surge cuando se generan instancias de poder no entre, sino por encima de los Estados. Se trata de entes a los que se cede soberanía, y que pueden ejercer dicha soberanía de manera autónoma en el territorio y sobre la población de los Estados, incluso ante la negativa de éstos GRIMM (2011: pp. 14-15). Es así que Stone SweEt y SAndHoltz (1997: p. 304) definen la supranacionalidad como una forma de poder en la que «centralized governmental structures (those organizations constituted at the supranational level) possess jurisdiction over specific policy domains within the territory comprised by the member states. In exercising that jurisdiction, supranational organizations are capable of constraining the behavior of

${ }^{1}$ Sin embargo, también hay que considerar que en la práctica existen supuestos de ámbitos de poder internacionales que se someten a complejos juegos de equilibrio y de neutralización mutua entre Estados que derivan en situaciones de autonomía fáctica de determinados centros de poder generando una dimensión supranacional. 
all actors, including member states, within those domains». El concepto de organización supranacional al que se está haciendo referencia es el previsto implícitamente en el art. 93 de la Constitución Española (organización o institución que ejerce competencias derivadas de la Constitución). En efecto, en los ámbitos de su competencia, la organización supranacional despliega una summa potestas, lo que supone que ejerce un poder soberano (y por lo tanto, derivado de la Constitución). Éste es el caso de la UE, tal y como se expresaba ya en 1963 el Tribunal de Justicia de las Comunidades Europeas en el asunto Van Gend en Loos, donde se señalaba que el Tratado de la Comunidad Económica Europea operaba «la creación de órganos en los que se institucionalizan poderes soberanos» ${ }^{2}$.

Si la organización supranacional cuenta con summa potestas y ejerce un poder que se impone sobre sus Estados miembros (afectando al territorio y los ciudadanos de éstos) incluso sin su consentimiento ${ }^{3}$, cabe preguntarse dónde se sitúan los límites conceptuales que la separan de la federación, o cuanto menos, de la confederación. A este respecto, podemos sugerir algunas líneas conceptuales en sus fundamentos más básicos. Con respecto a la federación el límite puede estar más o menos claro: mientras que la organización supranacional fundamenta su poder en la mera agregación de los poderes soberanos de sus Estados miembros, el Estado federal fundamenta su poder sobre sus Estados miembros y sobre un pueblo que sirve de sustrato unificador de la soberanía. Con respecto a la confederación, entidad política que fundamenta su poder exclusivamente en sus Estados miembros sobre la base de un pacto suscrito entre los mismos, el límite es más difuso MCCORMICK (2002: p. 6). A nuestro modo de ver, habría que utilizar como elemento diferenciador el carácter funcional (materialmente acotado) o territorial (generalista) del poder ejercido por la Organización ${ }^{4}$.

Una organización supranacional de carácter funcional nunca debería confundirse con una confederación, que es una instancia de poder de carácter generalista y base territorial. En ese caso, ¿cabría considerar la UE, que tiene carácter generalista, como una confederación? La UE constituye un género único de entidad política, puesto que puede concebirse como una instancia supranacional de poder territorial (única instancia de poder supranacional de carácter territorial en Derecho comparado): es decir, ejerce un poder que se extiende, en un territorio, sobre múltiples materias funcionales ${ }^{5}$. Por lo tanto,

2 STJCE, de 5 de febrero de 1963, as. 26/62 Van Gend en Loos, Rec. 1963, p. 339.

3 Es decir, la UE ejerce poderes soberanos conforme a la definición de BODIN (1992: p. 23) de soberanía como «power [...] of giving law to subjects in general without their consent».

${ }^{4}$ Sobre las categorías de gobernanza funcional y gobernanza territorial, ver HooGHE Y MARKS (2003).

${ }_{5}^{5}$ En este sentido, Grimm (2011: p. 17). 
en principio la UE constituiría una confederación. Sin embargo hay que atender también, desde una perspectiva más política, al hecho de que la confederación constituye más bien un ente político «de transición», ubicado en un proceso de construcción (o, en su caso, destrucción) nacional. Si se considera esto último, la asimilación de la UE a una confederación, aun siendo todavía posible, sería menos evidente 6 .

El paradigma de la soberanía estatal se ha proyectado históricamente en dos planos: summa potestas en el plano interno, e independencia en el plano internacional. De manera consecuente, la teoría clásica de las Relaciones Internacionales concebía a los Estados como entes autónomos, que no tenían ninguna esfera de poder por encima. Éste era al menos el esquema de sociedad internacional westfaliano. Sin embargo, los procesos de mundialización y globalización de los problemas han conllevado un aumento de las interdependencias entre los Estados, lo que ya de facto ha venido a reducir su independencia. En un primer momento los nuevos problemas se enfrentaron siguiendo los esquemas heredados del sistema westfaliano, pero a partir del siglo XX comienza a cambiar esta pauta y se crean dinámicas institucionales y comunitarias REUTER (1987: pp. 17-37). El paradigma relacional mantenía un sentido puro de la soberanía estatal: no se creaban estructuras superiores a los Estados para hacer frente a los problemas comunes sino que los Estados se coordinaban entre sí. Esta situación cambia con el carácter estructural de los problemas internacionales, que llamaba a la creación de organismos permanentes. Estos organismos permanentes se basaban inicialmente en una lógica internacional relacional, en la cual las organizaciones internacionales carecían de funcionarios y fondos propios (ambos recursos eran proporcionados por los Estados miembros), viendo los Estados salvaguardada su soberanía con un derecho de veto a la hora de tomar decisiones (que se adoptaban por unanimidad).

Sin embargo, esta situación comenzó a evolucionar a partir de una dinámica en la que los problemas internacionales se hacen tan urgentes y complejos que la comunidad internacional tiene que crear instancias de poder con suficiente agilidad operativa que escapen de los lentos mecanismos deliberativos de carácter intergubernamental, y que superen la posibilidad del bloqueo institucional por la acción de Estados no cooperativos. Surge así el modelo de organización supranacional.

Estructuras de poder supranacional, siempre en ámbitos sectoriales (es decir, funcionalmente acotados), pueden considerarse en el plano universal

${ }^{6}$ A este respecto, cabe mencionar a BuRgESs (2000: p. 49): «Our theoretical analysis suggests that the EC/EU is neither a federation nor a confederation in the classical sense. But it does claim that the European political and economic elites have shaped and moulded the EC/EU into a new form of international organization, namely, a species of «new» confederation.» 
la Organización Mundial del Comercio y la propia Organización de las Naciones Unidas (en lo concerniente al mantenimiento de la paz y la seguridad internacionales); y en el plano regional el Tribunal Europeo de Derechos Humanos del Consejo de Europa. Aunque estas organizaciones internacionales siguen obedeciendo, en sus fundamentos originarios, a tratados internacionales (lógica relacional), su desarrollo se independiza de la voluntad nacional, construyendo categorías jurídicas particulares que se imponen a los Estados.

La constitución de una esfera de poder autónomo que se sitúe por encima de los Estados y que se imponga a los mismos viene dada, en primer lugar, por mecanismos que hacen que el poder ejercido por el ente supranacional escape de la esfera de control de los Estados. Esto puede venir dado por elementos como la concesión de autonomía operativa a una esfera institucional (como la Comisión Europea, o el Parlamento Europeo) o la generación de mecanismos de toma de decisiones por mayoría cualificada de los Estados miembros (con lo que los Estados pierden su derecho de veto) ${ }^{7}$. En lo relativo a la concesión de autonomía operativa a una esfera institucional, cabe señalar determinadas cuestiones como la dotación de una personalidad internacional, el establecimiento de un funcionariado internacional, la concesión de privilegios e inmunidades, y sobre todo la creación de un presupuesto propio, que no dependa de las contribuciones de los Estados. En lo relativo a la autonomía operacional la importancia del poder presupuestario acaba resultando crucial.

En segundo lugar, junto con la autonomía, la supranacionalidad implica imposición de las políticas emanadas de la esfera supranacional sobre los Estados miembros. Esto se consigue a través de mecanismos como la dotación de eficacia directa y primacía a las normas y actos emanados de esa esfera institucional. La diferencia radical con las anteriores dinámicas intergubernamentales es que ahora a partir del plano supranacional se puede operar directamente y de manera autónoma en el plano nacional, subnacional y privado, sin que el Estado participe como «guardián»o pantalla.

Todas las características señaladas como propias de la supranacionalidad están presentes en el caso de la UE: por ello decimos que la UE es una entidad supranacional. Sin embargo, esas características no se presentan con igual fuerza en todas sus áreas competenciales. Existen áreas donde su poder político responde más a un modelo de organización internacional, es decir, donde prima la intergubernamentalidad sobre la supranacionalidad. En dichas áreas (como la Política Exterior y de Seguridad Común) el impulso político es en realidad la proyección de impulsos políticos nacionales agregados, que se fun-

\footnotetext{
${ }^{7}$ En este sentido, Poiares Maduro (2004: p. 13).
} 
den en la UE como si de un crisol se tratase. La supranacionalidad en la UE se extiende sobre ámbitos competenciales estrictamente delimitados. Supone así un poder soberano que se impone a los Estados, pero que sólo se extiende a los específicos ámbitos en los que los Estados, en el ejercicio de su soberanía, han decidido que así sea. Como es bien sabido, la UE carece de la KompetenzKompeten ${ }^{8}$, y por tanto, aunque ejerce poderes soberanos, no es en sí un sujeto soberano.

El fenómeno de la generación de mecanismos de control del poder supranacional ha sido identificado como un nuevo constitucionalismo (KUMM 2011), aunque quizás de manera precipitada. Lo cierto es que toda forma racional de poder político cuenta con mecanismos de control, ya sean explícitos o implícitos, positivizados o no, y la mera existencia de mecanismos de control no puede identificarse con una forma de constitucionalis$\mathrm{mo}^{9}$. A este respecto hay que considerar que en rigor, las instancias de poder supranacional no se sostienen sobre una Constitución, sino sobre un «proceso de constitucionalización»: así, habrá organizaciones supranacionales cimentadas sobre fundamentos más o menos constitucionalizados en función del grado en que se sostengan sobre estructuras normativas originarias que sean expresión de un poder soberano democráticamente legitimado. Esta proposición que sostenemos, sin embargo, es más dogmática que empírica, en la medida en que en la práctica actores como el Tribunal Constitucional Alemán han venido a rechazar esta lógica, refugiándose, ante el desconcierto provocado por la complejidad normativa y política de la construcción europea, en la reconfortante certeza de categorías añejas como nación, para, a través de la constatación de la inexistencia de una nación europea (tanto en su sentido de etnos, al no haber homogeneidad cultural; como en su sentido de demos, al no haber existido una voluntad constituyente democráticamente expresada ni unas narrativas políticas comunes) negar de entrada las posibilidades de un constitucionalismo supranacional LADEUR (1997: pp. 36-37), adoptando la postura holística del «todo o nada» definida por WALKER (2008: p. 524).

Sin embargo, lo cierto es que por la vía de hecho las nuevas formas de poder político supranacional parecen introducirnos en una era de post-constitucionalidad, en la que las instituciones trascienden el debate de constitución sí - constitución no, para situarse en un espectro gradual de progresiva

${ }^{8}$ Como se argumentaba de forma completa y detallada en la Decisión del TC Alemán relativa el Tratado de Maastricht, respecto al art. F. 3 del Tratado de Maastricht, así como en la Decisión del TC Alemán relativa al Tratado de Lisboa, respecto a los arts. 311 (que retomaba el art. F.3 de Maastricht) y 352 TFUE.

${ }^{9}$ Como señala Polares Maduro (2004: p. 4), una desustanciación del concepto de constitucionalismo como mera forma de organizar un poder puede llevarse al extremo de considerar que los estatutos de un club de golf son expresión de una constitución. 
cimentación constitucional, en el que se pueden alcanzar distintos grados de «constitucionalización». WALKER (2008: pp. 525-536) señala cinco elementos a través de los que se articula el encuadramiento constitucional del poder político, y que podemos concebir como ejes a lo largo de los cuales se produce la progresiva «constitucionalización»: 1. Existencia de un ordenamiento jurídico (omnicomprensivo, autointerpretativo, autónomo, autointegrado, autoejecutivo, autoenmendable, autodisciplinado); 2. Existencia de un marco institucional efectivo de órganos políticos con equilibrio de poderes; 3 . Conexión genética con un poder constituyente (que dota de legitimidad de origen); 4. Existencia de una comunidad política integrada, sujeto y objeto de la soberanía (que dota de legitimidad de ejercicio); 5. Existencia de una lógica discursiva que sigue un parámetro binario de constitucionalidad/inconstitucionalidad.

Los cinco ejes definidos, no obstante, pueden resultar incompletos conforme a determinadas concepciones del constitucionalismo como consagración de los valores superiores de una sociedad. A este respecto cabe recordar la triple distinción que hace PoIARES MADURO (2004: p. 4) del constitucionalismo como 1) mera forma de limitación del poder; 2) plasmación de los valores superiores de la sociedad (en el sentido del modelo de «constitución constitucional» del art. 16 de la Declaración de los Derechos del Hombre y del Ciudadano ${ }^{10}$ ); y en medio de esas dos categorías, 3) marco de deliberación para fijar el interés público. El constitucionalismo al que se refieren los cinco elementos de encuadramiento identificados por Walker es un constitucionalismo que no necesariamente ha de plasmar los valores superiores de la sociedad, y que podría ubicarse en la categoría intermedia, como marco de deliberación para la fijación del interés público. Es de subrayar el hecho de que esa forma de constitucionalismo supone una aspiración hacia un interés público, lo que implica un interés común. Un interés que va más allá de los intereses particulares y que por lo tanto, implica la existencia de un centro de poder superior. Lo que quiere decir que esta forma de constitucionalismo podrá plantearse en las organizaciones supranacionales, y no así en las internacionales, toda vez que en estas últimas no existe propiamente un interés común, sino una agregación de los intereses de los Estados miembros.

Para que puedan plantearse los elementos de constitucionalización, primero tenemos que constatar la existencia de un centro de poder autónomo, que impacte directamente en los Estados y sus ciudadanos. La supranacionalidad constituye por tanto un prius lógico para que pueda plantearse la constitucionalización. En la UE, de acuerdo con su naturaleza de «Comunidad de Derecho» (en expresión de Walter HaLlsteIN), se ha producido una evolución institucional en la que los avances hacia la supranacionali-

10 «Toda sociedad en la cual la garantía de los derechos no esté asegurada, ni la separación de poderes determinada, carece de Constitución». 
dad han ido acompañados de avances hacia el constitucionalismo. Es decir, que es un poder que ha venido siendo embridado por un marco de progresiva cimentación constitucional ${ }^{11}$. Una cimentación constitucional que ha servido no sólo para controlar, sino también para legitimar un poder supranacional que, en ausencia de una clara legitimidad de origen constituyente, y con una precaria base democrática, ha buscado asideros donde sustentar una difícil y siempre sutil legitimidad.

Esta cimentación constitucional se puede comprobar en elementos como la cohesión proporcionada al sistema normativo comunitario por el Tribunal de Justicia de la Unión, el diseño institucional construido sobre un modelo de división y equilibrio de poderes, y el asentamiento democrático de la Unión. Un asentamiento democrático que se expresa a través de circuitos de autorización, representación y participación, que proporcionan legitimidad de origen $^{12}$; así como de circuitos de transparencia, rendición de cuentas y capacidad de respuesta, que proporcionan legitimidad de ejercicio.

La existencia de un poder presupuestario propio viene a constituir no sólo un prius lógico para la operatividad del constitucionalismo más allá del Estado, sino que también constituye una base tanto para la existencia de dinámicas de equilibrio de poderes como para el reforzamiento de las dinámicas de transparencia y rendición de cuentas. En efecto, la previsión constitucional de un poder ejecutivo que gasta (art. 311 del Tratado de Funcionamiento de la Unión Europea - TFUE) y un legislativo que autoriza (art. 314 TFUE) y controla (art. 319 TFUE), con la ayuda de una Entidad de Fiscalización Superior (arts. 285-287 TFUE), en el contexto de una relación de confianza parlamentaria (que se sustanciará en la investidura y, en su caso, en una moción de censura - arts. 17 del Tratado de la Unión Europea (TUE) y 234 TFUE), tiende a mantener un equilibrio de poder constitucional propio de una forma de gobierno parlamentaria. De la importancia que el poder presupuestario ha tenido en la articulación de estos mecanismos de «pesos y contrapesos» da muestra el contexto en el que se produjo la dimisión de la Comisión Santer en 1999.

Igualmente, las previsiones relativas tanto a la programación presupuestaria como al control presupuestario realizado por el Tribunal de Cuentas Europeo y el Parlamento Europeo tienden a reforzar la transparencia y la rendición de cuentas en el ejercicio del poder financiero de la Unión. Como señaló el ECOFIN en sus conclusiones de 16 de marzo de 2010 (sobre el presupuesto de 2011), el presupuesto europeo es «one of the most significant tools to guarantee the accountability of the European Union towards its citi-

${ }^{11}$ Poiares Maduro (2004: p. 11): «In the process of European integration, constitutionalism as the form of power followed the claim of normative authority".

12 A través de mecanismos como las elecciones al Parlamento Europeo, el derecho de petición, la asistencia del Defensor del Pueblo, o la iniciativa legislativa popular. 
zens (...); an accurate and accountable use of the EU resources is one of the essential means to reinforce the trust of the European citizens».

Por lo tanto, el poder presupuestario de la UE puede ser considerado tanto una condición previa como un elemento de desarrollo en el constitucionalismo europeo. A continuación vamos a analizar en detalle en qué medida el poder presupuestario de la UE ha contribuido a configurar un poder supranacional y cómo ha favorecido simultáneamente al encuadramiento constitucional de dicho poder.

\section{LA PROGRAMACIÓN PRESUPUESTARIA EUROPEA}

La UE puede considerarse en términos jurídicos como una asociación voluntaria de Estados establecida mediante acuerdo internacional y dotada de órganos permanentes, propios e independientes, encargados de gestionar unos intereses colectivos y capaces de expresar una voluntad jurídicamente distinta de la de sus miembros. Es decir, responde al patrón específico de un tipo de sujeto de Derecho Internacional, que en doctrina iusinternacionalista se llama organización internacional (SOBRINO HEREDIA 2003: p. 43), independientemente de que su poder sea internacional o supranacional.

Como toda instancia de poder, en principio la UE surge para gestionar unos intereses colectivos de relevancia política. En efecto, para que exista una involucración a nivel inter o supranacional, los problemas que se planteen tienen que generar externalidades que vayan más allá del concreto ámbito estatal. En el caso de la UE existen determinados problemas políticos que hacen necesaria la creación de programas de gasto público supranacionales. Es el supuesto, por ejemplo, de la política de cohesión, que pretende el desarrollo económico territorial. Esto tiene externalidades a nivel supranacional en la medida en que el desarrollo económico «equilibrado y sostenible» ${ }^{13}$ tiene un impacto directo sobre la distribución de rentas y sobre el mercado interior de toda la UE. Lo cual genera la necesidad de una involucración a nivel supranacional a través del gasto público. Es el caso igualmente de otras políticas sectoriales como agricultura, como investigación y desarrollo tecnológico, o como la política de cooperación al desarrollo; todas las cuales se proyectan a través de programas de gasto.

El Estado constitucional se sustenta sobre el control del poder a partir del Derecho. El poder estatal puede expresarse en la práctica a través de múltiples formas: legislación y regulación, intervención directa, certificación, canalización de información... así como gasto público. Sobre todo, podemos

${ }_{13}$ Art. 3.3 TUE: «La Unión establecerá un mercado interior. Obrará en pro del desarrollo sostenible de Europa basado en un crecimiento económico equilibrado [...]. La Unión fomentará la cohesión económica, social y territorial y la solidaridad entre los Estados miembros». 
decir, gasto público: porque el Estado social, forma en la que se constituyen la mayoría de los Estados del mundo desarrollado, articula gran parte de su actividad en torno al gasto público. Resulta por lo tanto muy difícil imaginar el ejercicio del poder público en un marco constitucional sin la existencia de un sistema de ordenación de los ingresos y gastos públicos consistente.

La UE es una organización supranacional: lo que implica que opera como una instancia de poder autónoma que en su desarrollo se independiza de la voluntad de los Estados miembros, construyendo categorías jurídicas particulares que se imponen a los Estados mismos incluso en contra de su voluntad. Una de las características de su naturaleza supranacional sería la posibilidad de contar con un presupuesto dotado de recursos propios derivados de una autoridad presupuestaria propia. Un presupuesto dotado de recursos propios incide tanto en la autonomía de la organización supranacional (que deja de depender de contribuciones nacionales) como en la superación de la soberanía estatal (al poder exigir en Derecho el pago de impuestos tanto a entes nacionales como subnacionales o privados, aun en contra de la voluntad del Estado).

Del carácter nuclear que tanto la dimensión supranacional como la categoría de recursos propios tienen para la UE da cuenta el hecho de que ambos conceptos ya quedasen reflejados en el Tratado de París de 1951. En efecto, en el art. 9 de dicho Tratado se hacía referencia al carácter supranacional de la Alta Autoridad, y en su art. 78 se introducía la expresión de recursos propios. Una expresión que posteriormente quedaría definida con su significado actual en el Tratado de la Comunidad Económica Europea de 1957, en cuyo art. 201 se señalaba que «La Comisión estudiará en qué condiciones las contribuciones financieras de los Estados miembros [...] podrían ser sustituidas por recursos propios, especialmente por ingresos procedentes del arancel aduanero común, tras el establecimiento de éste».

En 1965, un primer intento de establecer un sistema de recursos propios mediante la transferencia de los derechos de aduana y exacciones agrícolas (recursos procedentes de las políticas comunitarias aduanera y agrícola) se vio frustrado frente a la oposición de la Francia de Charles de Gaulle ${ }^{14}$. No fue sino hasta la cumbre de La Haya de 1969 que se acordó desbloquear este avance, y en 1970, el Consejo adoptó una decisión por la que se asignaban a las Comunidades (con un presupuesto único tras el Tratado de fusión de $1965)$ recursos propios para cubrir todos sus gastos. La decisión marcó el fin del sistema de contribuciones nacionales.

Actualmente el sistema de recursos propios tiene como primer fundamento el art. $311 \mathrm{TFUE}^{15}$. Sin embargo, la proclamación normativa que hace di-

${ }^{14}$ Lo cual contribuyó a la «crisis de la silla vacía» de 1965 y al Compromiso de Luxemburgo de 1966.

15 «La Unión se dotará de los medios necesarios para alcanzar sus objetivos y para llevar a cabo sus políticas. Sin perjuicio del concurso de otros ingresos, el presupuesto 
cho artículo tiene que ser matizada. En efecto, puede considerarse que la realidad del presupuesto europeo lo aproxima en la práctica más a un modelo presupuestario propio de una organización internacional convencional de carácter intergubernamental BERNICOT (2004: p. 76) ${ }^{16}$.

\subsection{El sistema de recursos propios}

Jurídicamente, el significado de un sistema de recursos propios implica que los recursos que se transfieren a la UE son propios de la UE. No son pues recursos de los ciudadanos de la Unión o de los Estados miembros que éstos transfieren a la UE, sino que los fondos le pertenecen a la UE desde el momento en que se produce el «hecho impositivo» correspondiente. Específicamente, se dice que los recursos propios son «debidos en Derecho» a la UE, lo que implica que la transferencia de su propiedad no requiere ninguna decisión jurídica por parte de los Estados miembros, sino que es «automática», aun con la eventual intermediación de éstos a efectos de transferir la posesión sobre los fondos (conforme a los arts. 1 y 2 del Reglamento 1150/2000 ${ }^{17}$ ). En caso de que los Estados miembros no transfieran la posesión de los fondos conforme a los procedimientos previstos, el art. 11 del Reglamento 1150/2000 establece la posibilidad de exigir intereses de demora ${ }^{18}$.

La importancia de un sistema de recursos propios para el desarrollo coherente de las políticas públicas puede comprobarse en la propia historia del constitucionalismo contemporáneo, manifestándose concretamente en el proceso constitucional norteamericano. En efecto, uno de los fallos que más se le achacó a los Artículos de la Confederación fue la falta de reconocimiento de la facultad del gobierno central para establecer y recaudar impuestos (UROFSKY y FinKELMANN 2002: pp. 81-82). En virtud de los Artículos, los recursos financieros eran aportados por los estados miembros. En esta situación, el Congreso se veía obligado a depender completamente de los gobiernos de sus estados miembros, que decidían si pagaban o no las cantidades requeridas por el Congreso. Ciertos estados decidían no pagar las contribu-

será financiado íntegramente con cargo a los recursos propios».

16 « Les finances publiques européennes s'apparentent plus au mécanisme de financement et de dépenses d'organismes de coopération multilatérales qu'aux finances publiques des États membres ».

${ }_{17}$ Artículo 1: Los recursos propios de las Comunidades [...] serán puestos a disposición de la Comisión y se controlarán en las condiciones establecidas en el presente Reglamento [...] Artículo 2: [...] un derecho de las Comunidades sobre los recursos propios [...] se constatará cuando se cumplan las condiciones previstas por la reglamentación aduanera en lo relativo a la consideración del importe del derecho y su comunicación al deudor.

18 «Todo retraso en las consignaciones en la cuenta mencionada en el apartado 1 del artículo 9 dará lugar al pago de intereses [...]». 
ciones, o las pagaban sólo en parte. Sin una fuente constante de ingresos suficientes para hacer cumplir sus leyes y tratados suscritos, o pagar sus deudas, y sin un mecanismo para obligar a los Estados a pagar, la Confederación quedaba en una situación vulnerable. Fue esto precisamente lo que motivó el reconocimiento, en la Constitución de 1787, de la capacidad del Estado federal de recabar impuestos (la taxing and spending clause $^{19}$, reconocida en la sección 8 del artículo I, que permitía establecer, recabar y gastar impuestos).

El sistema de recursos propios de la UE se articula en torno a un presupuesto, que recoge todos los ingresos previstos y gastos autorizados para el periodo de un año. En teoría, en el derecho presupuestario europeo rige el principio de universalidad, por el cual se incluyen todos los ingresos y todos los gastos; en la práctica, permanecen fuera del presupuesto los recursos del Fondo Europeo de Desarrollo, los recursos del Banco Europeo de Inversiones, así como las operaciones de préstamo que pueda realizar la Unión como acreedora.

En 2013 el presupuesto de la UE preveía un gasto de 150.900 millones de euros en créditos de compromiso, lo que suponía el 1,13\% de la Renta Nacional Bruta (RNB) del conjunto de la Unión. Este presupuesto se financia mediante un sistema de recursos propios dividido en un $70 \%$ de fondos aportados por los Estados miembros y calculados en proporción de su RNB respectiva, un $12 \%$ de derechos de aduana y exacciones agrícolas y un 10,5\% procedente de una proporción sobre el Impuesto sobre el Valor Añadido (IVA) recabado por los Estados miembros. Finalmente, existe una «quinta categoría» residual de recursos (que supone alrededor de un 7,5\% del presupuesto), tales como los ingresos por multas impuestas en el marco de políticas de la Unión, ingresos por intereses de demora, los impuestos a funcionarios europeos, los ingresos por venta de bienes y servicios producidos por las propias Instituciones de la Unión (como publicaciones), y las contribuciones de terceros países a ciertos programas de la Unión (como determinados programas de Investigación y Desarrollo Tecnológico).

En teoría, el poder presupuestario expresado en los presupuestos anuales de la Unión recae de manera conjunta sobre el Consejo (instancia de poder intergubernamental) y el Parlamento (instancia de poder supranacional), con preponderancia de este último. En la práctica, el presupuesto europeo se encuentra predeterminado por el «marco financiero plurianual», que «tendrá por objeto garantizar la evolución ordenada de los gastos de la Unión dentro del límite de sus recursos propios» (art. 312 TFUE). La adopción de marcos financieros plurianuales (inicialmente llamados «perspectivas financieras») constituye una práctica impuesta por la necesidad de mantener el principio de equilibrio financiero del art. 310.1 TFUE. En efecto, los marcos financieros plurianuales co-

19 «The Congress shall have Power To lay and collect Taxes, Duties, Imposts and Excises, to pay the Debts and provide for the common Defence and general Welfare of the United States». 
menzaron a adoptarse en 1988, en un momento de crecimiento vertiginoso de los gastos de la Unión. Hasta el momento se han adoptado cinco marcos financieros: Delors I (1988-1992), Delors II (1993-1999), Agenda 2000 (20002006), el marco 2007-2013 y el actual marco financiero plurianual 2014-2020. Las cuatro primeras «perspectivas financieras» se adoptaron sobre la base de mecanismos sui generis, con carácter más político que jurídico, Acuerdos Inter-Institucionales entre el Parlamento, el Consejo y la Comisión. El peso del acuerdo recaía sobre el Consejo, en la medida en que debía pronunciarse por unanimidad. Con el Tratado de Lisboa este procedimiento viene a positivizarse constitucionalmente, siendo incorporado en el art. 312 TFUE como uno de los «procedimientos legislativos especiales» donde el Consejo se pronuncia por unanimidad; en este caso, previa aprobación del Parlamento. El art. 312 TFUE prevé una duración mínima de cinco años para el marco financiero, aunque en la práctica su duración se ha mantenido en siete años ${ }^{20}$.

El marco financiero plurianual fija los importes de los límites máximos anuales de créditos para compromisos, por categoría de gastos, así como el límite máximo anual de créditos para pagos, para todo el presupuesto (art. 312.2 TFUE). Por lo tanto, el marco financiero plurianual queda referenciado a las categorías de gastos que han de corresponder a los grandes sectores de actividad de la Unión.

Respecto a la definición de categorías de gastos realizada por el marco financiero plurianual, hay que señalar que, si bien alrededor de un $80 \%$ del presupuesto europeo se gasta en los fondos estructurales y en los fondos agrícolas, existen más de 65 programas de gasto de pequeña dimensión (menos de 1.000 millones de euros al año), gestionados en su mayor parte de manera centralizada por la Comisión (sobre todo programas pertenecientes a las áreas de «Libertad, Seguridad y Justicia» y «Ciudadanía»). Sólo 11 programas, que reciben más de 1.000 millones de euros al año, aglutinan el 94\% del presupuesto. La disgregación del presupuesto en una multitud de programas de baja cuantía desemboca en una estructura de «espolvoreado presupuestario» (saupoudrage budgetaire), en la que el gasto de la Unión difícilmente alcanza a tener un impacto visible a nivel transnacional europeo (CIPRIANI 2010: p. 18).

En cuanto a la determinación de los ingresos de la Unión, el sistema de recursos propios presenta un sesgo intergubernamental todavía más acusado. En el ya mencionado art. 311 TFUE se señala que «La Unión se dotará de los medios necesarios para alcanzar sus objetivos y para llevar a cabo sus políticas», y a dichos efectos se prevé otro «procedimiento legislativo especial» para la adopción de «Decisiones Presupuestarias». En este procedimiento

${ }^{20}$ La previsión de que tenga cinco años de duración es una cláusula que puede adquirir sentido en la medida en que se haga coincidir la duración del marco financiero con el mandato quinquenal del Parlamento (y de la Comisión). 
legislativo especial el Consejo se pronuncia por unanimidad, y el Parlamento sólo ha de ser consultado previamente, pronunciándose mediante un Dictamen no vinculante. El establecimiento o supresión de categorías de recursos propios sólo entra en vigor una vez que la Decisión Presupuestaria haya sido aprobada por los Estados miembros de conformidad con sus respectivas normas constitucionales (art. 311 TFUE y art. 11 de la Decisión 2007/436, del Consejo), lo que ha llevado a que parte de la doctrina considere que estas Decisiones del Consejo son asimilables de facto a «Derecho originario» (CIPRIANI 2010: p. 99).

A lo largo de la historia de la UE se han adoptado seis Decisiones Presupuestarias; la actual Decisión Presupuestaria cubría el periodo del marco financiero plurianual 2007-2013 (Decisión 2007/436, del Consejo), y sigue manteniéndose bajo el marco 2014-2020, aunque el Parlamento y el Consejo acordaron en junio de 2013 crear un grupo de alto nivel para estudiar una nueva Decisión Presupuestaria. Además de la tipología de los recursos que han de financiar el presupuesto europeo y las pautas de repartición de las cargas entre los Estados miembros, la Decisión Presupuestaria establece un techo máximo de recursos para la UE, que para el marco financiero 2007-2013 nunca podrá superar el 1,24\% de la RNB de la Unión. El calado político de las Decisiones Presupuestarias es muy grande, ya que en ellas se dibujan las categorías de contribuyentes y receptores netos al presupuesto (lo que desde la opinión pública se percibe como los «ganadores y perdedores» de la lucha intergubernamental de poder en el seno de la Unión).

La primera Decisión Presupuestaria fue adoptada en 1970, y a partir de ella comenzó a construirse el sistema de recursos propios. Dicho sistema de recursos propios había sido previsto en el ya mencionado art. 201 del Tratado de la Comunidad Económica Europea para cuando entrase en funcionamiento la Unión Aduanera (hito que se alcanzó en 1968); con anterioridad a esa fecha las Comunidades Europeas habían sido financiadas conforme a un sistema de contribuciones estatales, en base a un reparto fijo establecido en el art. 200 del Tratado de la Comunidad Económica Europea ${ }^{21}$. Entre 1970 y 1975 se produce la transición hacia un sistema de recursos propios.

${ }^{21}$ Artículo 200. 1. Los ingresos del presupuesto comprenderán, aparte de otros ingresos, las contribuciones financieras de los Estados miembros, determinadas con arreglo a la clave de reparto siguiente: Bélgica // 7,9 Alemania // 28 Francia // 28 Italia // 28 Luxemburgo // 0,2 Países Bajos // 7,9

2. No obstante, las contribuciones financieras de los Estados miembros destinadas a sufragar los gastos del Fondo Social Europeo se determinarán con arreglo a la clave de reparto siguiente: Bélgica // 8,8 Alemania // 32 Francia // 32 Italia // 20 Luxemburgo // 0,2 Países Bajos // 7

3. Las claves de reparto podrán ser modificadas por el Consejo, por unanimidad. 
De la misma forma que inicialmente el sistema presupuestario de los Estados Unidos se nutría de contribuciones nacionales, en la UE hasta 1970 los ingresos comunitarios estaban compuestos también por contribuciones nacionales, que servían para financiar un pequeño presupuesto europeo compuesto básicamente por los gastos de funcionamiento y los fondos agrícolas. A partir de 1970, el sistema de recursos propios comunitarios prevé tres categorías: los llamados recursos propios tradicionales (integrando exacciones agrícolas y derechos de aduana), y los recursos basados en el IVA. El cuarto recurso, basado en una proporción de la RNB de los Estados miembros, se añadió en 1988.

Las exacciones agrícolas se recaudan de manera análoga a los aranceles, sobre las importaciones agrícolas de países terceros en relación con productos incluidos en una Organización Común de Mercado (strictu sensu son aranceles que recaen sobre una categoría de importaciones determinada). Tienen por función compensar la diferencia entre el precio mundial de los productos y el precio superior garantizado a los agricultores europeos ${ }^{22}$. Los derechos de aduana por su parte derivan de la aplicación a las importaciones de los correspondientes aranceles exteriores comunes.

En cuanto al recurso del IVA, es una exacción aplicada sobre las bases imponibles globales del IVA de cada Estado miembro, con una revisión anual referenciada a los ingresos netos que ese Estado miembro haya recaudado en concepto de IVA. Esta revisión existe debido a que la proporción del consumo en las rentas de los países es distinta, cumpliéndose la ley económica por la cual los países más pobres tienden a tener un mayor peso del consumo en relación con su renta, lo que produciría -en ausencia de corrección- que los países más pobres pagarían proporcionalmente más que los ricos, constituyendo un supuesto de imposición regresiva que podría ser contrario a los objetivos de cohesión económica y territorial enunciados en el art. 3 TUE.

Finalmente, el recurso procedente de la RNB fue introducido por el Consejo Europeo de Bruselas en 1988 y supone una imposición a los Estados miembros basada en la RNB de cada uno de ellos y conforme a un tipo variable cada año según las necesidades presupuestarias. El recurso RNB completa a los restantes recursos propios para alcanzar el nivel de gastos presupuestado en cada año, de conformidad con el principio de equilibrio presupuestario fijado en el art. 310.1 TFUE. De ahí que su montante varíe todos los años (compensando las posibles variaciones que experimenten los otros recursos debido a la coyuntura económica). A pesar de su carácter

${ }^{22}$ Se incluye también en este apartado una categoría especial, las cotizaciones del azúcar, establecidas en el marco de la organización del mercado del azúcar y de la isoglucosa para autofinanciar dicho mercado, mediante cotizaciones a la producción y almacenamiento. 
inicialmente complementario y residual, la evolución del sistema financiero europeo, marcada por una falta de sentido de la supranacionalidad y el egoísmo de los Estados miembros, ha hecho que este recurso se convierta en el recurso con mayor peso presupuestario: en 2013 financia alrededor del $74 \%$ del presupuesto.

\subsection{Análisis del carácter supranacional del sistema de recursos propios}

El sistema de recursos propios puestos a disposición de la Unión, que se supone un rasgo más del carácter supranacional de la UE, tiene un escoramiento intergubernamental tan grande que difícilmente se puede hablar de que en realidad existan unos recursos auténticamente «propios» de la Unión. En primer lugar hay que considerar que la Unión carece de autonomía para establecer sus propios recursos financieros, que son en la práctica fijados por los Estados miembros. Los procedimientos de decisión para la determinación de los ingresos y los gastos de la Unión en los marcos financieros plurianuales arriba mencionados son procedimientos intergubernamentales, y la necesidad de tomar las Decisiones Presupuestarias por unanimidad genera dinámicas de «egoísmo» que desembocan en un modelo de recursos presupuestarios intergubernamental.

En este contexto la expresión «recursos propios» no deja de ser una entelequia, puesto que la relación directa entre el sujeto contribuyente y la UE es bastante débil en el caso de las exacciones y aranceles, y en los recursos derivados del IVA y la RNB es meramente simbólica. En la práctica, la noción de recursos propios sólo quiere decir que son recursos debidos por derecho a la Unión. En el ámbito presupuestario la UE se fundamenta sobre una retórica que tiene poco correlato con la realidad de hecho.

Los recursos propios existentes sólo son «propios» en cuanto a su nombre, puesto que constituyen en realidad contribuciones nacionales fijadas a través de complejos mecanismos. De las cuatro categorías de recursos propios previstas por el Derecho financiero de la Unión, sólo las exacciones agrícolas y los derechos de aduana serían auténticamente «impuestos europeos», satisfechos por los ciudadanos (y aun así no son satisfechos directamente, puesto que se trata de impuestos indirectos, que además son recaudados por las autoridades nacionales). El recurso procedente del IVA es proporcionado por los Estados miembros sobre una base armonizada del IVA nacional, mientras que el recurso de RNB es proporcionado también por los Estados miembros en proporción a su RNB: ambos recursos no son más que contribuciones nacionales calculadas conforme a parámetros variados.

El sistema de recursos propios habría de implicar que los ingresos no fuesen contribuciones que hacen los Estados al presupuesto de la Unión, sino contribuciones que hacen los propios ciudadanos. Sin embargo, el supuesto 
de relación directa entre ciudadanos y presupuesto europeo no se da completamente, ya que no existe un servicio recaudador europeo análogo a las Administraciones Tributarias de las haciendas estatales, sino que la percepción corresponde a los Estados miembros. De acuerdo con el Reglamento 2891/1977, los Estados miembros transfieren los tributos a las arcas de la Comunidad en un plazo máximo de dos meses $^{23}$, limitándose la Comisión a controlar que las sumas recaudadas sean correctas.

El hecho de que el sistema de recursos propios venga referenciado a unos recursos directamente relacionados con los Estados miembros (proporción de RNB, proporción del IVA), atiza el discurso de las balanzas fiscales (expresión de la diferencia entre la contribución total que desde un Estado miembro se hace a la Unión, y las transferencias que desde la Unión se hacen a dicho Estado), lo que implica que se perciba desde la opinión pública el carácter de Estados contribuyentes y receptores netos como equivalente a los «ganadores y perdedores» de la lucha intergubernamental de poder en el seno de la Unión. Subyacente a esta idea se sitúa la misma lógica que concibe que el contribuyente europeo no es en rigor el propio ciudadano, sino más bien los diversos Estados, y que lo que para la Unión constituye un ingreso en realidad para el Estado representa un gasto.

En este contexto, el interés de los Estados miembros consiste en inclinar lo máximo posible a su favor la respectiva balanza fiscal (un interés que tenderá incluso a primar sobre la consecución de los objetivos políticos transnacionales que se persiguen con los programas de gasto europeos). La presión intergubernamental en la lucha por las balanzas fiscales conduce a que el sistema de ingresos de la Unión no sea un sistema uniforme, sino que esté sujeto a múltiples exenciones, regímenes especiales, «cheques nacionales», compensaciones, etc., siguiendo una lógica intergubernamental de negociaciones y pactos transaccionales ${ }^{24}$.

El propio presupuesto europeo no es un instrumento uniforme, puesto que existen excepciones al principio de no compensación (es decir, fondos preasignados a determinados gastos, como es el caso de la contribución financiera de los Estados miembros a algunos programas de investigación), así como fondos extrapresupuestarios (como el Fondo Europeo de Desarrollo, o los

${ }^{23}$ Reteniendo, en el caso de los aranceles y exacciones agrícolas, un 25\% del montante total percibido, en concepto de gastos de gestión.

${ }^{24}$ A este respecto ilustra CIPRIANI (2010: p. 101): «the UK rebate (which limits the balance between UK payments to the EU budget and EU expenditure in its favour to onethird), the variable financing of this rebate according to the member states concerned (Germany, Austria, the Netherlands and Sweden pay only 25\% of their normal share), the limitation of the VAT resource calculation base to $50 \%$ of GNI, the reduction of the rate of call of the VAT resource for some member states (Austria, Germany, the Netherlands and Sweden), the member states' levy of $25 \%$ of customs duties collected and ad hoc 'bonuses'for several member states». 
fondos manejados por el Banco Europeo de Inversiones ${ }^{25}$. La consiguiente falta de respeto a los principios de universalidad y de no compensación amplifica la opacidad del presupuesto, lo que va en detrimento de la conexión con los subsistemas de control político y social. Además, la multiplicidad de programas de gasto de escasa cuantía hace que muchos de ellos no puedan tener un impacto visible a nivel transnacional europeo (CIPRIANI 2010: p. 18), lo que socava la proyección del poder supranacional.

El problema del sistema presupuestario de la Unión no sólo va en el sentido de la contradicción que supone llamar «recursos propios» a lo que en realidad son contribuciones intergubernamentales; también es notable el problema de la complejidad del sistema. En efecto, el ciudadano europeo no sabe cuánto paga, lo que contribuye a un desinterés en lo relativo a la buena gestión financiera de la Unión, socavándose el control social, cúspide del sistema de control presupuestario. Como señala SHAH (2004: p. 38), la disociación entre decisiones de imposición y decisiones de gasto perjudica la correcta rendición de cuentas del sector público. Por otro lado, la concepción del Estado miembro como sujeto de Derecho financiero de la Unión puede ser más o menos cierta a nivel del ingreso (recordemos, alrededor del $90 \%$ de los ingresos de la Unión pueden considerarse contribuciones de los Estados), pero no al nivel del gasto. Los beneficiarios de los principales programas de subvenciones de la Unión son los ciudadanos, no los Estados miembros directamente. Todo ello conduce a una disociación entre el debate sobre los ingresos de la Unión, que se hace a nivel de los Estados miembros, y el debate sobre la asignación de los gastos, donde tienen más preponderancia los ciudadanos. Esto puede llevar a que se pida mucho (porque no se es consciente del coste de los programas de gasto, ya que los ciudadanos en la práctica no contribuyen directamente al presupuesto de la Unión), y que se fiscalice socialmente poco (igualmente, porque no se es consciente del coste que para el bolsillo del contribuyente tienen las políticas de la Unión), lo cual acaba derivando en un sistema de control presupuestario excesivamente enfocado hacia la espectacularidad mediática (por lo tanto, obsesionado con la incidencia del fraude), y guiado más bien por dinámicas políticas de cuestionamiento del propio proyecto europeo (euroescepticismo).

La situación del sistema impositivo europeo resulta por lo tanto paradójica. Como señala CIPRIANi (2010: pp. 66-67), si en 1776 los colonos america-

${ }^{25}$ Todo esto ha llevado al Parlamento Europeo a lamentar que en la evolución del sistema financiero de la Unión, los regímenes de excepción de los que va acompañado el sistema de recursos propios «han aumentado su complejidad, lo han hecho más oscuro para los ciudadanos y cada vez menos equitativo, y han dado lugar a un sistema de financiación que ha provocado desigualdades inaceptables entre los Estados miembros» (Parlamento Europeo, Resolución de 8 de junio de 2005 sobre los retos políticos y medios presupuestarios de la Unión ampliada 2007-2013, párr. L). 
nos se quejaban de que debían hacer frente a impuestos sin tener la posibilidad de verse políticamente representados, en 2014 los ciudadanos europeos se ven políticamente representados, pero no perciben que tengan impuestos a los que hacer frente. El principio «no taxation without representation», en su formulación de «no representation without taxation» no se da en el caso de la Unión: hay representación en el seno del Parlamento Europeo, pero no hay una tributación visible y explícita (CIPRIANI 2007: p. 63) ${ }^{26}$. La falta de una relación directa entre ciudadano y presupuesto europeo es una manifestación más del «déficit de legitimidad democrática» de la Unión (MORAVCSIK 2002; FOLLESDAL Y HIX 2005), que en último término socava la solidez constitucional de la construcción europea.

En definitiva, a pesar de la retórica de los «recursos propios», puede afirmarse que el ámbito de las finanzas públicas de la Unión constituye una de las esferas donde las dinámicas intergubernamentales son más fuertes. Se comprueba así cómo las finanzas públicas constituyen un elemento nuclear de los Estados y por ello éstos se resisten a transferir el ejercicio de competencias financieras a la Unión.

\section{CONCLUSIONES}

En el presente trabajo hemos defendido un concepto de supranacionalidad que se referencia a la soberanía propia del Estado. En este ámbito semántico, el concepto de organización supranacional hace referencia a instancias de poder autónomas, que pueden tomar decisiones que se imponen a los Estados incluso ante la negativa de éstos. Las organizaciones supranacionales suponen por tanto organizaciones o instituciones que ostentan el ejercicio de competencias soberanas, derivadas de la Constitución (como se prevé en el art. $93 \mathrm{CE}$ ). La cesión del ejercicio de competencias soberanas se lleva a cabo precisamente en un contexto en el que los problemas políticos que se plantean sólo pueden hacerse frente a través de acciones y políticas cuya dimensión y efectos desbordan y trascienden las capacidades de los Estados (como se prevé en el art. 5.2 TFUE).

La supranacionalidad se erige en fundamento lógico para concebir la existencia de un constitucionalismo más allá del Estado. Siguiendo a Walker, hemos considerado que el encuadramiento constitucional del poder supranacional

${ }^{26}$ Es así que el Parlamento Europeo se expresaba en 1994 señalando: «the transfer to the Community of an increasing number of political powers in fields such as agricultural policy, external trade, internal trade, development aid, transport and social policy and economic and monetary policy should go hand in hand with corresponding action to provide the Union with democratic legislative powers, which would include both tax and budgetary powers and appropriate financial autonomy ('no representation without taxation')» (European Parliament, Resolution of 21 April 1994, para. 21). 
se articulará y desarrollará a lo largo de cinco ejes: 1 . Un ordenamiento jurídico (omnicomprensivo, autointerpretativo, autónomo, autointegrado, autoejecutivo, autoenmendable, autodisciplinado); 2. Un marco institucional efectivo de órganos políticos con equilibrio de poderes; 3 . Una conexión genética con un poder constituyente (que dota de legitimidad de origen); 4. Una comunidad política integrada, sujeto y objeto de la soberanía (que dota de legitimidad de ejercicio); 5 . Una lógica discursiva que sigue un parámetro binario de constitucionalidad/inconstitucionalidad. En el caso de la UE, se comprueba la existencia de un poder supranacional, que pretende fundamentarse en unos cimientos constitucionales propios, más allá de los propios cimientos constitucionales de sus Estados miembros. Esta cimentación constitucional europea se observa en todos los puntos de encuadramiento constitucional identificados, menos en el $3^{\circ}$ (al no existir una conexión genética con un poder constituyente propio). Así se pueden identificar elementos como el Derecho originario y derivado de la Unión (que aspira a configurar un «ordenamiento jurídico» propio $^{27}$ ); la cohesión proporcionada a dicho sistema normativo por el Tribunal de Justicia de la Unión, que además actúa como jurisdicción constitucional validando los actos de la Unión en función de su compatibilidad o incompatibilidad con el Derecho originario; el diseño institucional construido sobre un modelo de división y equilibrio de poderes; así como el asentamiento parcialmente democrático de las Instituciones de la Unión.

En este contexto, la existencia de un poder presupuestario supranacional europeo no se limita a constituir un prius lógico para la operatividad de un constitucionalismo europeo, proyectando políticas y acciones que podrán ser en último término juzgadas siguiendo la lógica binaria de constitucionalidad/ inconstitucionalidad. El presupuesto de la Unión va más allá, y pretende ofrecer una base tanto para la potenciación de dinámicas de equilibrio de poderes (con un poder ejecutivo que gasta y un legislativo que autoriza y controla) como para el reforzamiento de las dinámicas democráticas de transparencia y rendición de cuentas (a través del control presupuestario).

Sin embargo, en lo relativo a la configuración de la ciudadanía europea no ya como sujeto, sino como objeto de la soberanía, hemos comprobado cómo los Estados siguen actuando como pantallas que se interponen entre la Unión y los propios ciudadanos, interfiriendo en la proyección hacia los ciudadanos del poder financiero de la Unión, lo cual distorsiona la dimensión supranacional en este ámbito y menoscaba el encuadramiento constitucional del poder de la Unión. Siguiendo una constante histórica en la que los recursos financieros se presentan como elemento nuclear del poder estatal, los Estados miembros de la Unión se han guardado mucho de transferir el ejercicio de la soberanía en este ámbito. El escoramiento intergubernamental resultante no deja de ser un ele-

27 STJCE, de 15 de julio de 1964, as. 6/64 Costa c. Enel, Rec. 1964, p. 2258. 
mento preocupante en una Unión caracterizada por la descoordinación de las políticas fiscales nacionales y la incidencia de crisis asimétricas, y aquejada por fuertes desequilibrios en la distribución territorial de rentas.

\section{BIBLIOGRAFÍA}

BERNICOT, JEAN-FrançOIS (2004): La place et la représentation des finances publiques dans l'Union européenne. Revue Française de Finances Publiques. 84.

BoDIn, JEAN (1992): On sovereignty, Cambridge University Press.

Burgess, Michael (2000): Federalism and European union: The building of Europe, 1950-2000. Routledge.

CIPRIANI, G. (2007): Rethinking the EU Budget: Three Unavoidable Reform, Bruselas, CEPS Paperbacks.

(2010): The EU Budget Responsibility without Accountability?, Bruselas, CEPS Paperbacks.

FOLLESDAL Y HIX (2005): Why there is a democratic deficit in the EU: A response to Majone and Moravcsik. European Governance Papers (EUROGOV), No. C-05-02.

GrIMM, DieTER (2011): The Achievement of Constitutionalism. En The Twilight of Constitutionalism? Oxford University Press.

Hooghe, L., \& Marks, G. (2003): Unraveling the Central State, but How? Types of Multi-level Governance. American Political Science Review, 97(2).

Kumm, Mattias (2011): The Best of Times and the Worst of Times, en The Twilight of Constitutionalism? Oxford University Press.

LADEUR, K. (1997): Towards a Legal Theory of Supranationality - The Viability of the Network Concept. European Law Journal, 3.

Loughlin, Martin (2011): What is Constitutionalism? En The Twilight of Constitutionalism? Oxford University Press.

McCORMick, John P. (2002): Understanding the European Union, Palgrave McMillan.

Moravcsik, A. (2002): In Defence of the «Democratic Deficit»: Reassessing the Legitimacy of the European Union. Journal of Common Market Studies, Vol. 40, 4.

Poiares Maduro, Miguel. (2004): How Constitutional Can the European Union $B e$ ? The Tension Between Intergovernamentalism and Constitutionalism in the European Union, en Weiler and Eisgruber, eds., Altneuland: The EU Constitution in a Contextual Perspective, Jean Monnet Working Paper 5/04.

Reuter, Paul (1987): Derecho internacional público, Barcelona, Bosch.

SHAH, ANWAR (2004): Fiscal decentralization in developing and transition economies: progress, problems, and the promise, Policy Research Working Paper Series 3282, The World Bank.

Stone SweEt, A., SANDHOltz, W. (1997): European integration and supranational governance. Journal of European public policy, 4(3).

Urofsky, Melvin I.; PAUl Finkelman (2002): A March of Liberty: A Constitutional History of the United States 1 (2nd ed.). New York, NY: Oxford University Press. WAlKer, NeIL. (2008): Taking Constitutionalism beyond the State, Political Studies, 56. 
TITLE: The constitutional dimension of the European Union budgetary power.

RESUMEN: En el contexto del debate sobre las transformaciones que está experimentando el constitucionalismo contemporáneo, un lugar prominente viene a ser ocupado por la extensión de marcos constitucionales a realidades sociopolíticas que van más allá del Estado. El presente artículo pretende indagar en la morfología de estas nuevas realidades objeto del constitucionalismo, utilizando el especifico caso de la Unión Europea y su poder presupuestario como evidencia empírica sobre la que explorar los contornos conceptuales del constitucionalismo supranacional. Pretende, básicamente, responder a dos cuestiones: ¿qué es la supranacionalidad y qué relación tiene este concepto con el constitucionalismo?, y ¿en qué medida el poder presupuestario europeo configura un poder supranacional inscrito en un marco constitucional?

El artículo se estructura en torno a tres apartados. En primer lugar, se realiza una aproximación teórica al constitucionalismo supranacional. En segundo lugar, se describe el sistema presupuestario europeo, que pasará a ser analizado a través del concepto de supranacionalidad. Finalmente, la última sección resume las proposiciones formales y empíricas más relevantes.

PALABRAS CLAVE: Unión Europea (UE), Supranacionalidad, Constitucionalismo, Presupuesto europeo.

ABSTRACT: In the context of the debate on the changes that contemporary constitutionalism is experiencing, a prominent place is occupied by the extension of constitutionalism to sociopolitical realities that go beyond the state. This article aims to investigate the sociopolitical morphology of these new constitutional realities, using the specific case of the European Union (EU) and its budgetary power as an empirical lens through which to explore the conceptual contours of supranational constitutionalism. It aims basically to answer two questions: what is supranationality and what relation does this concept have with respect to constitutionalism? And, to what extent does the European budget reflect a supranational power inscribed within a constitutional framework?

The article is structured around three sections. First, it takes a theoretical approach to supranationality. Secondly, it describes the European budgetary system, which will be analyzed through the concept of supranationality, pondering the extent to which it responds to a supranational power scheme. Finally, the last section summarizes the most relevant formal and empirical propositions raised.

KEY WORDS: European Union (EU), Supranationality, Constitutionalism, EU budget. 